\title{
Cambios cinemáticos de la marcha en pacientes con artrosis de rodilla con diferentes descargas de peso
}

\author{
Kinematic Changes of Gait in Patients with Knee Osteoarthritis by Different Weight bearings \\ Mudanças cinemáticas da marcha em pacientes com artrose de joelho com diferentes descargas de peso \\ Margareth Lorena Alfonso-Mora FT MsC, ${ }^{1}$ Adolfo Ávila-Barón Ing MsC2 \\ Recibido: 28 de mayo de $2013 \bullet$ Aceptado: 20 de junio de 2014 \\ Doi: dx.doi.org/10.12804/revsalud12.03.2014.02
}

Para citar este artículo: Alfonso-Mora ML, Ávila-Barón A. Cambios cinemáticos de la marcha en pacientes con artrosis de rodilla con diferentes descargas de peso. Rev Cienc Salud. 2014;12(3): 319-29. doi: dx.doi.org/10.12804/revsalud12.03.2014.02

\begin{abstract}
Resumen
Objetivo: Describir las diferencias en las variables cinemáticas de la marcha en mujeres mayores de 60 años con artrosis de rodilla con diferentes descargas de peso. Materiales y métodos: Diseño investigativo longitudinal Phanel con un muestreo no probabilístico a conveniencia en mujeres $\mathrm{n}=16$, con edad > 60 años, sobrepeso y artrosis de rodilla; el control de la descarga de peso se realizó por medio de una plataforma de rehabilitación locomotora midiéndose la marcha en tres momentos con el $100 \%$, $90 \%$ y $80 \%$ del peso. Resultados: Los rangos de movimiento para algunas fases de la marcha fueron diferentes para la descarga de peso utilizadas $\mathrm{p}<0,05$; para la velocidad y aceleración angular no se encontraron diferencias estadísticamente significativas. Conclusiones: Las diferentes descargas de peso no representaron para la presente investigación una variable que modificara de forma significativa las variables cinemáticas de la marcha.
\end{abstract}

Palabras clave: Osteoartritis, marcha, aceleración.

\section{Abstract}

Objective: To describe the differences in kinematic gait variables in women over sixty years of age with knee osteoarthritis by different weight bearings. Material and Methods: Phanel longitudinal research design with convenience sampling probabilistic $n=16$ women, aged $>60$ years, obesity and knee osteoarthritis, control weight bearing was performed by a locomotive rehabilitation platform that measured gait three times with weights of $100 \%, 90 \%$ and $80 \%$. Results: The movement ranges in certain gait phases were different for bearings of used weight $\mathrm{p}<0.05$; for

1 Universidad de La Sabana. Correspondencia: margarethalmo@unisabana.edu.co

2 Universidad Santo Tomás de Tunja. 
angular speed and acceleration no statistically significant differences were found. Conclusions: The different weight bearings for this research represented a variable that significantly change the kinematic gait variables.

Key Words: Osteoarthritis, Gait, Acceleration.

\section{Resumo}

Objetivo: descrever as diferenças nas variáveis cinemáticas da marcha em mulheres maiores de sessenta anos com artrose de joelho com diferentes descargas de peso. Materiais e métodos: concepção investigativa longitudinal Phanel com uma amostragem não probabilística a conveniência em mulheres $n=16$ com idade $>60$ anos, sobrepeso e artrose de joelho, o controle da descarga de peso se realizou por meio de uma plataforma de reabilitação locomotora medindo-se a marcha em três momentos com o 100\% do peso, 90 e 80. Resultados: Os rangos de movimento para algumas fases da marcha foram diferentes para a descarga de peso utilizada $\mathrm{p}<0.05$, para a velocidade e aceleração angular não se encontraram diferenças estatisticamente significativas. Conclusões: As diferentes descargas de peso não representaram para a presente pesquisa uma variável que modificará de forma significativa as variáveis cinemáticas da marcha.

Palavras-chave: Osteoartrite, Marcha, Aceleração.

\section{Introducción}

La artrosis de rodilla es una enfermedad de alta prevalencia en los adultos mayores de 60 años, especialmente de género femenino, entidad que cursa con signos y síntomas que limitan funcionalmente a los pacientes y trae consigo modificaciones estructurales y funcionales en los miembros inferiores que infieren en el patrón de marcha $(1,2)$. Los pacientes con artrosis de rodilla, disminuyen la cadencia, la longitud de paso y de zancada, aumentan el ancho de paso, disminuyen la velocidad lineal, angular $\mathrm{y}$, a su vez, la aceleración (2).

La transmisión de cargas cambia en todo el tren inferior durante la marcha, la cadera se alinea en rotación externa durante el contacto inicial y la rodilla tiende a aumentar su angulación en varo (3); por su parte, en el tobillo aumenta la supinación, se adelanta la transmisión de carga durante el contacto inicial y disminuye la alineación metatarsal; la suma de estas modificaciones trae consigo un patrón de marcha más lento y menos funcional (4).

El diagnóstico de artrosis usualmente está acompañado por sobrepeso y obesidad; esta condición debe ser controlada por medio de ejercicio y una dieta con baja ingesta calórica para contribuir a la disminución del peso corporal y traer consigo una baja en el dolor y las limitaciones funcionales. De esta manera, se evidencia una relación entre la obesidad y las complicaciones sintomatológicas en esta articulación que incluye también cambios en la marcha (5).

Se ha descrito cómo se comportan las variables cinemáticas y se ha demostrado que existe una relación entre la disminución de la velocidad de la marcha y los rangos de movilidad de la rodilla, con mayor proporción al momento de extenderla (6). Adicionalmente, se reporta que las anomalías mecánicas que 
acompañan la artrosis de la articulación de la rodilla en una fase temprana pueden tener implicaciones etiológicas, pero puede que otros representen cambios secundarios desarrollados en parte como mecanismo de compensación en la artrosis de rodilla; dentro de algunos cambios se encuentra: mayor angulación en varo y rotación interna durante la fase de apoyo y de impulsión, durante el momento extensor la respuesta de carga se incrementa en la parte medial de la rodilla lo que hace la transmisión de carga ineficiente y dolorosa (7).

Los estudios que muestran los cambios en la marcha en pacientes con artrosis de rodilla también exponen la existencia de modificaciones en los componentes cinéticos y cinemáticos de la marcha; además, sustentan cambios en los determinantes y fases de la misma pero no reportan el comportamiento de esta con diferentes descargas de peso. Entonces, nace la necesidad de conocer cuál es la conducta de las variables cinemáticas de la misma con diferentes descargas de peso, además de observar la respuesta sintomatológica de la articulación. Por lo tanto, el objetivo de la presente investigación es describir los cambios en las variables cinemáticas de la marcha en mujeres mayores de 60 años con artrosis de rodilla con diferentes descargas de peso.

\section{Materiales y métodos}

El diseño de investigación utilizado fue Longitudinal Phanel, siendo un solo grupo de participantes observadas en tres momentos de tiempo diferentes, correspondientes a la suspensión en la plataforma de rehabilitación locomotora con el $100 \%$, el $90 \%$ y el $80 \%$ del peso corporal, fundamentándose en la hipótesis de establecer la diferencia en los rangos de movimiento, velocidad, aceleración angular y síntomas de dolor en las diferentes fases de la marcha, en cada descarga de peso.
Se conformó una muestra de 16 mujeres utilizándose un muestreo no probabilístico a conveniencia. Los criterios de inclusión fueron: diagnóstico de artrosis de rodilla confirmado con radiografía, sobrepeso u obesidad, edad mayor a 60 años y género femenino; los criterios de exclusión fueron: signos vitales inestables, artrosis de cadera o tobillo, artrosis de rodilla estadio avanzado, índice de masa corporal por arriba de $34,9 \mathrm{~kg} / \mathrm{m}^{2}$ y alteraciones posturales que pudieran modificar de antemano el patrón de marcha. Se realizaron las mediciones previas al consentimiento informado a las participantes $\mathrm{y}$ al firmarlo.

El análisis videográfico se realizó por medio de cámaras JVC HD con frecuencia de registro de $48 \mathrm{kHz}$ en el plano sagital y el programa Kinovea setap. Primero, se hizo una historia clínica de cada participante y se registró: edad, antecedentes patológicos, estatura y peso; posteriormente, se colocaron cinco marcadores para análisis en el plano sagital de la siguiente manera: cabeza del quinto metatarsiano, talón, maléolo externo, cóndilo femoral externo y cabeza femoral. A continuación, se suspendieron a las pacientes del arnés para cuantificar la descarga de peso que se inició con el 100 \% del peso corporal, se utilizó una velocidad de 3,0 $\mathrm{km} / \mathrm{h}$ con inclinación de 0 , se hicieron cuatro tomas de video en movimiento con cada descarga de peso $(100 \%, 90 \%, 80 \%)$, analizándose la última por considerarse la más fisiológica.

Se realizó el cálculo de los rangos de movimiento en cada fase de la marcha en el plano sagital para miembros inferiores; se reprodujo cuadro a cuadro utilizando los marcadores previamente descritos como guías para determinar los rangos de movimiento implicados en cada fase; seguidamente, se calculó la velocidad angular y aceleración angular dedicando el tiempo calculado por el software durante el ciclo de la marcha. 
El control de la descarga de peso se realizó por medio de una plataforma de rehabilitación locomotora que contó con un subsistema de sensor tipo celda de carga, un motor DC con caja reductora 1:90, un sistema de poleas con una guaya para transmisión directa de la carga y un sistema electrónico de ajuste automático. La plataforma también tuvo un sistema inteligente embebido en un procesador digital de señales (DSP) alimentado con los valores análogos de voltaje de las variables, frecuencia cardiaca, grado del dolor expresado en forma digital del 0 a 10 por el paciente, el promedio de la descarga de peso obtenido en la celda de carga en un intervalo de tiempo; las salidas por la realización de nuevos ajustes en la descarga de peso.

Se realizó un análisis estadístico descriptivo de valores de tendencia central y de dispersión por medio de la desviación estándar, así como pruebas estadísticas para determinar las diferencias significativas en los tres momentos de evaluación de la marcha, por medio de cálculos de modelos lineales generales para medidas repetidas con la prueba Bonferroni con una significancia del 0,05, situación realizada en las ocho fases de la marcha en cada articulación del miembro inferior, esto en el programa SPSS versión 11.0.

\section{Resultados}

\section{Análisis descriptivo}

Se examinaron 16 mujeres con los criterios de inclusión mencionados, el promedio de edad fue de 63 años +3 , la media en el peso corporal fue de 69 $\mathrm{kg}+7 \mathrm{~kg}$, el índice de masa corporal fue de 30,29 +4 , lo que indica que la mayoría de las mujeres examinadas presentan sobrepeso u obesidad. La media del peso controlado por medio de la plataforma de rehabilitación locomotora con el $80 \%$ fue de $13,8 \mathrm{~kg}$; el control del peso con el $90 \%$ fue de $7 \mathrm{~kg}$; la media de la frecuencia cardiaca en reposo fue de $75+10$ latidos por minuto; por su parte, la frecuencia cardiaca de trabajo fue de $130+24$ latidos por minuto, la velocidad utilizada en la plataforma de rehabilitación fue constante de $3 \mathrm{~km} / \mathrm{h}$.

Con respecto al dolor, se encontró un promedio de $3+2$, medido con la escala análoga verbal del dolor para los tres momentos de medición, esta se realizó antes, durante y después de la prueba. Es claro que la sintomatología no se modifica con la descarga de peso, pues la diferencia de medias entre los tres momentos de evaluación del dolor no fue estadísticamente significativa $\mathrm{p}>0,05$.

\section{Análisis diferencial}

Al realizar la comparación entre los promedios de rangos de movimiento en el tobillo, en las diferentes fases con las tres descargas de peso, en el contacto inicial, apoyo medio, apoyo final, y prebalanceo existen diferencias significativas; en el contacto inicial, al comparar el $80 \%$ con el $100 \%$ del peso corporal, la situación coincide con la fase de apoyo final y prebalanceo; a su vez, en el apoyo medio, cuando se compara el $90 \%$ con el $100 \%$, también se evidencian diferencias estadísticamente significativas (tabla 1 ); sin embargo, al realizar un análisis gráfico de los promedios es claro que el rango de movimiento con respecto al $100 \%$ de la carga es diferente en cada fase de la marcha (figura 1).

La comparación de promedios con respecto al rango de movimiento en la rodilla, en las diferentes fases de la marcha, fue diferente en el apoyo final al comparar específicamente el $90 \%$ con el $100 \%$, en el resto de variables (figura 2), la significancia estadística no muestra que las diferencias sean representativas en los tres momentos de evaluación para el rango de movimiento de la articulación en mención. La significancia estadística de la totalidad de las fases de la marcha en los tres momentos 
Tabla1. Diferencias estadísticas del rango de movimiento en grados

\begin{tabular}{|c|c|c|c|c|c|c|c|}
\hline & $\dot{\mathrm{x}} 80$ & DS & $\dot{\mathrm{x}} 90$ & DS & $\dot{\mathrm{x}} 100$ & DS & Sig. \\
\hline \multicolumn{8}{|l|}{ Tobillo } \\
\hline Contacto inicial & 90,4 & 4,7 & 88 & 4 & 87,1 & 2,9 & $0,007^{* *}$ \\
\hline Respuesta a la carga & 89,3 & 4 & 89,7 & 4,1 & 88 & 3,7 & $>0,05$ \\
\hline Apoyo medio & 87,8 & 5,4 & 89,0 & 3,2 & 86,5 & 3,4 & $0,050^{*}$ \\
\hline Apoyo final & 90,1 & 3,3 & 89,8 & 5,4 & 85,6 & 4,8 & $0,015^{*}$ \\
\hline Prebalanceo & 100 & 5,5 & 100,8 & 5,3 & 95,3 & 3,6 & $0,010^{*}$ \\
\hline Balanceo inicial & 96,6 & 5,9 & 97,5 & 5,5 & 94,5 & 3 & $>0,05$ \\
\hline Balanceo medio & 90,6 & 6,2 & 90,5 & 4,1 & 90,4 & 3,3 & $>0,05$ \\
\hline Balanceo final & 89,2 & 4,3 & 89,3 & 3,4 & 89,4 & 3,5 & $>0,05$ \\
\hline \multicolumn{8}{|l|}{ Rodilla } \\
\hline Contacto inicial & 161 & 16 & 159,5 & 13,6 & 160,8 & 13,2 & $>0,05$ \\
\hline Respuesta a la carga & 162 & 10 & 162,8 & 8,2 & 161,8 & 9,8 & $>0,05$ \\
\hline Apoyo medio & 167 & 9 & 162,3 & 12,4 & 167,8 & 6 & $>0,05$ \\
\hline Apoyo final & 172 & 4,5 & 168,4 & 4,7 & 173 & 5,3 & $0,020^{*}$ \\
\hline Prebalanceo & 151,6 & 16,2 & 150 & 11,7 & 160,4 & 12 & $>0,05$ \\
\hline Balanceo inicial & 135 & 7 & 128,6 & 9,8 & 133 & 8,4 & $>0,05$ \\
\hline Balanceo medio & 129,8 & 6,4 & 130,1 & 9,5 & 130,5 & 7,2 & $>0,05$ \\
\hline Balanceo final & 160,9 & 16,3 & 160,2 & 14,2 & 160,3 & 14,3 & $>0,05$ \\
\hline \multicolumn{8}{|l|}{ Cadera } \\
\hline Contacto inicial & 155,6 & 6,1 & 155 & 4,5 & 154,3 & 5,8 & $>0,05$ \\
\hline Respuesta a la carga & 168,3 & 6,3 & 169 & 4,7 & 167,5 & 5,7 & $>0,05$ \\
\hline Apoyo medio & 177,5 & 7,2 & 174 & 6,2 & 178 & 5 & $>0,05$ \\
\hline Apoyo final & 194,9 & 4,7 & 191 & 5,6 & 193,8 & 5,6 & $>0,05$ \\
\hline Prebalanceo & 189 & 9,6 & 186,6 & 6,9 & 183,7 & 24,1 & $>0,05$ \\
\hline Balanceo inicial & 178 & 160 & 175,8 & 5,6 & 179,3 & 5,71 & $>0,05$ \\
\hline Balanceo medio & 160,5 & 6,5 & 162,3 & 5,3 & 162,8 & 4,6 & $>0,05$ \\
\hline Balanceo final & 154 & 4,4 & 154,7 & 4,3 & 154,7 & 7,14 & $>0,05$ \\
\hline
\end{tabular}

* Significancia $\alpha=0,05$. Probado a través de pruebas para medidas repetidas de Bonferroni.

**Significancia $\alpha=0,01$ Probado a través de pruebas para medidas repetidas de Bonferroni.

de evaluación en la articulación de la cadera no estableció diferencias relevantes (tabla 1).

Posterior al análisis de la velocidad angular en la articulación del tobillo para la dorsiflexión y plantiflexión, durante las fases de la marcha, la significancia estadística de la mayoría de las fases de la marcha indicó que no son diferentes los promedios entre los tres momentos de evaluación; sin embargo, de la fase del apoyo final al prebalanceo en los tres momentos los promedios de la velocidad angular presentan diferencias significativas. La flexoextensión de la rodilla en los tres momentos de evaluación no son diferentes situación también dada para la articulación de la cadera (tabla 2). 
Alfonso-Mora ML, Ávila-Barón A

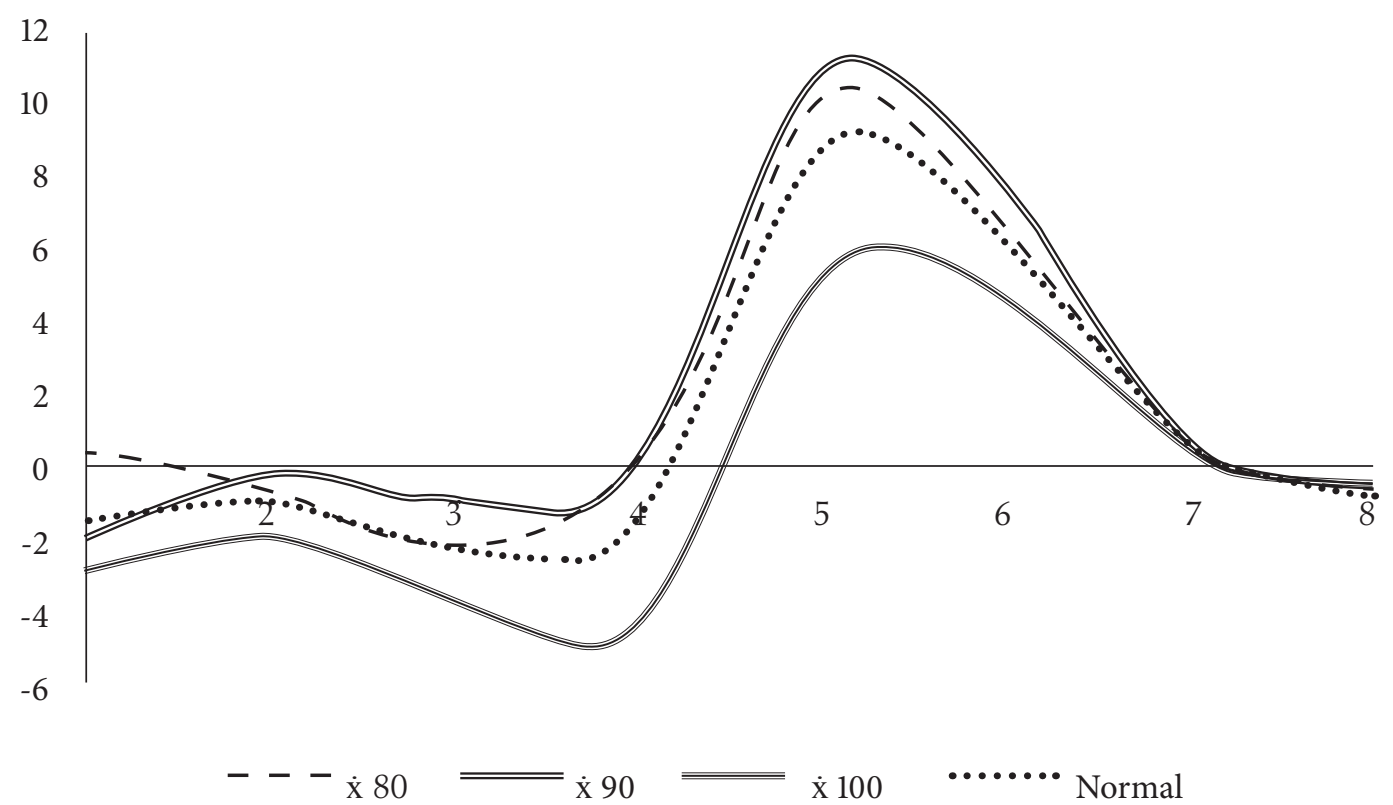

Figura 1. Rangos de Movimiento en el tobillo durante la marcha con Diferentes Descargas de Peso

*Diferencias en los rangos de movimiento con cada descarga de peso durante las fases de la marcha, en comparación con rangos de movimiento normales entiéndase 1 como contacto inicial y 8 como el balanceo final.

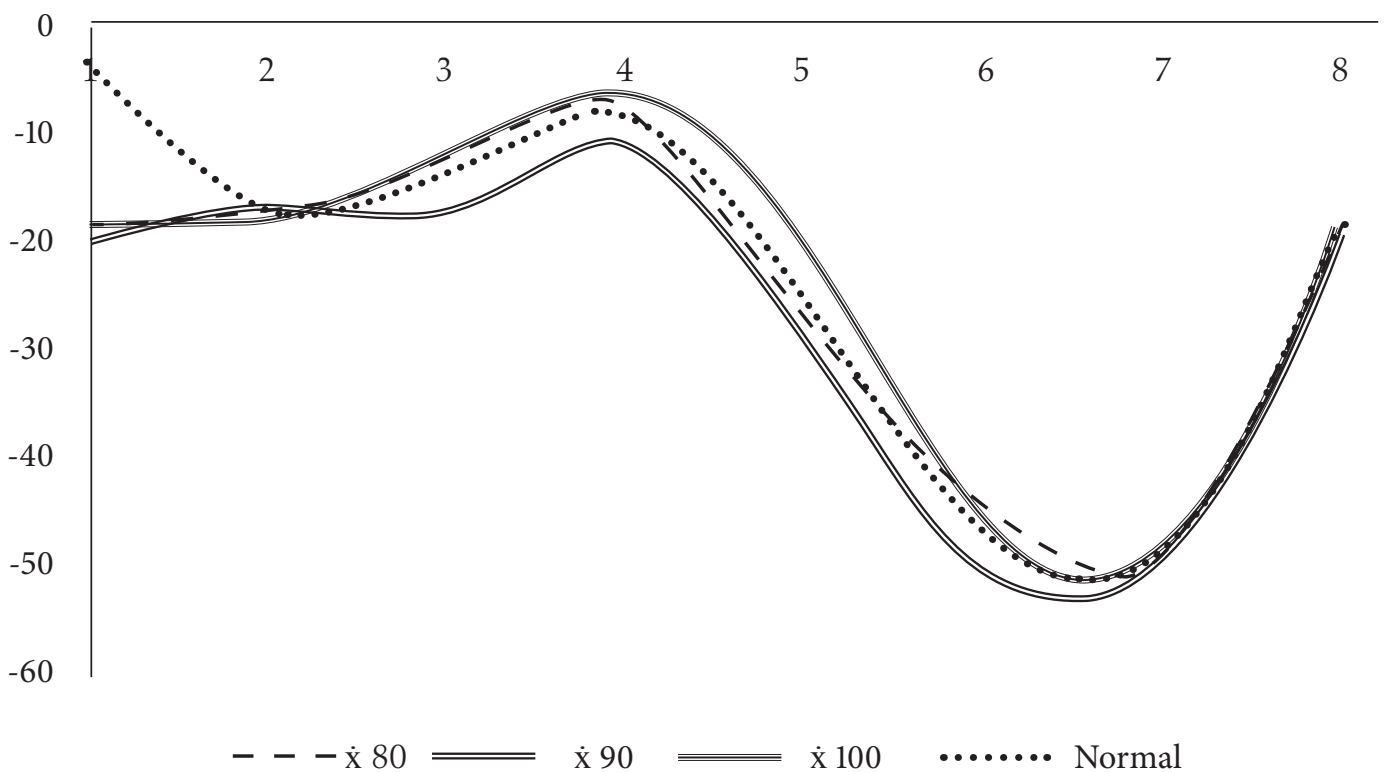

Figura 2. Rangos de Movimiento en Rodilla durante la marcha con Diferentes Descargas de Peso

*Diferencias en los rangos de movimiento con cada descarga de peso durante las fases de la marcha, en comparación con rangos de movimiento normales entiéndase 1 como contacto inicial y 8 como el balanceo final. 
Tabla 2. Diferencias estadísticas en la velocidad angular en grados/milisegundo

\begin{tabular}{|c|c|c|c|c|c|c|c|}
\hline & $\dot{\mathrm{x}} 80$ & DS & $\dot{\mathrm{x}} 90$ & DS & $\dot{\mathrm{x}} 100$ & DS & Sig. \\
\hline \multicolumn{8}{|l|}{ Tobillo } \\
\hline Contacto inicial a respuesta a la carga & $-0,562$ & 0,28 & $-0,11$ & 0,23 & $-0,043$ & 0,25 & $>0,05$ \\
\hline Respuesta a la carga a apoyo medio & 0,14 & 0,57 & 0,11 & 0,32 & 0,15 & 0,48 & $>0,05$ \\
\hline Apoyo medio a apoyo final & $-0,086$ & 0,19 & $-0,048$ & 0,23 & 0,033 & 0,19 & $>0,05$ \\
\hline Apoyo final a prebalanceo & $-0,71$ & 0,4 & $-0,77$ & 0,42 & $-0,69$ & 0,47 & $0,016^{*}$ \\
\hline Prebalanceo a balanceo inicial & 0,35 & 0,71 & 0,28 & 0,54 & 0,07 & 0,40 & $>0,05$ \\
\hline Balanceo inicial a balanceo medio & 0,60 & 0,53 & 0,75 & 0,49 & 0,41 & 0,42 & $>0,05$ \\
\hline Balanceo medio a balanceo final & 0,19 & 0,89 & 0,07 & 0,27 & 0,05 & 0,26 & $>0,05$ \\
\hline \multicolumn{8}{|l|}{ Rodilla } \\
\hline Contacto inicial a respuesta a la carga & $-0,015$ & 0,58 & $-0,17$ & 0,38 & $-0,05$ & 0,35 & $>0,05$ \\
\hline Respuesta a la carga a apoyo medio & $-0,55$ & 0,91 & 0,050 & 0,91 & $-0,60$ & 0,71 & $>0,05$ \\
\hline Apoyo medio a apoyo final & $-0,19$ & 0,38 & $-0,24$ & 0,55 & $-0,20$ & 0,35 & $>0,05$ \\
\hline Apoyo final a prebalanceo & 1,5 & 1,27 & 1,27 & 0,78 & 0,90 & 0,89 & $>0,05$ \\
\hline Prebalanceo a balanceo inicial & 1,66 & 1,75 & 2,14 & 0,80 & 2,73 & 0,75 & $>0,05$ \\
\hline Balanceo inicial a balanceo medio & 0,51 & 0,7 & $-0,13$ & 1,21 & 0,25 & 0,82 & $>0,05$ \\
\hline Balanceo medio a balanceo final & $-1,5$ & 0,59 & $-1,48$ & 0,64 & $-1,49$ & 0,62 & $>0,05$ \\
\hline \multicolumn{8}{|l|}{ Cadera } \\
\hline Contacto inicial a respuesta a la carga & $-0,63$ & 0,28 & $-0,71$ & 0,19 & $-0,65$ & 0,27 & $>0,05$ \\
\hline Respuesta a la carga a apoyo medio & $-0,92$ & 0,46 & $-0,54$ & 0,49 & $-1,050$ & 0,52 & $0.018^{*}$ \\
\hline Apoyo medio a apoyo final & $-0,66$ & 0,25 & $-0,67$ & 0,27 & $-0,60$ & 0,25 & $>0,05$ \\
\hline Apoyo final a prebalanceo & 0,42 & 0,57 & 0,36 & 0,43 & 0,71 & 1,83 & $>0,05$ \\
\hline Prebalanceo a balanceo inicial & 1,05 & 0,59 & 1,1 & 0,49 & 0,44 & 2,5 & $>0,05$ \\
\hline Balanceo inicial a balanceo medio & 1,79 & 0,64 & 1,3 & 0,61 & 1,6 & 0,40 & $>0,05$ \\
\hline Balanceo medio a balanceo final & 0,29 & 0,36 & 0,37 & 0,37 & 0,52 & 0,34 & $>0,05$ \\
\hline
\end{tabular}

*Sig. Significancia estadistica menor a 0,05 . Probado a través de pruebas para medidas repetidas de Bonferroni.

Con respecto a la aceleración angular, en la articulación del tobillo en cada fase de la marcha existen diferencias significativas, en la del apoyo medio al apoyo final, del mismo modo que del apoyo final al balanceo inicial y en el resto del ciclo de balanceo. Los cambios en la acele- ración angular en flexoextensión de la rodilla en todas las fases de la marcha no presentaron diferencias estadísticamente significativas; $\sin$ embargo, en la única fase de la marcha en que se evidenciaron fue en la de balanceo inicial a balanceo medio (tabla 3). 
Tabla 3. Diferencias estadísticas en la aceleración angular en grados/milisegundo

\begin{tabular}{|c|c|c|c|c|c|c|c|}
\hline & $\dot{\mathrm{x}} 80$ & DS & $\dot{\mathrm{x}} 90$ & DS & $\dot{\mathrm{x}} 100$ & DS & Sig. \\
\hline \multicolumn{8}{|l|}{ Tobillo } \\
\hline Contacto inicial a respuesta a la carga &,- 0075 & 01949 &,- 1267 & ,23212 &,- 0067 & ,02093 & $>0,05$ \\
\hline Respuesta a la carga a apoyo medio & ,0056 & 01861 & ,1133 & ,33566 & ,0040 & ,02028 & $>0,05$ \\
\hline Apoyo medio a apoyo final & 0169 & 01250 &,- 0077 & ,17151 &,- 0260 &, 01404 & $<0,001^{* *}$ \\
\hline Apoyo final a balanceo inicial &,- 0446 & ,04658 &,- 8095 & ,41092 &,- 0337 &, 03460 & $0.00^{*}$ \\
\hline Balanceo inicial a balanceo medio & 0175 & 03554 &, 2733 &, 55609 & ,0570 & ,02411 & $0.013^{*}$ \\
\hline Balanceo medio a balanceo final & ,0131 &, 04143 &, 7600 &, 51242 & ,0353 & ,02973 & $<0,001^{* *}$ \\
\hline \multicolumn{8}{|l|}{ Rodilla } \\
\hline Contacto inicial a respuesta a la carga & ,0153 & ,03603 &,- 0067 & ,03658 &, 0153 & ,02232 & $>0,05$ \\
\hline Respuesta a la carga a apoyo medio &,- 0080 & 02859 & ,0080 & ,04057 &,- 0087 & ,01846 & $>0,05$ \\
\hline Apoyo medio a apoyo final &,- 0440 &, 03641 &,- 0373 & 02987 &,- 0287 & 02748 & $>0,05$ \\
\hline Apoyo final a prebalanceo &,- 0003 & ,12313 &,- 0405 & ,05829 &,- 0744 &, 06557 & $>0,05$ \\
\hline Balanceo inicial a balanceo medio &, 0787 & ,08895 & ,1110 & ,03869 & ,1360 & 03911 & $>0,05$ \\
\hline Balanceo medio a balanceo final &, 0720 & 03167 &, 0447 & ,04912 & ,0587 & ,03815 & $>0,05$ \\
\hline \multicolumn{8}{|l|}{ Cadera } \\
\hline Contacto inicial a respuesta a la carga & ,0080 & ,02145 &,- 0067 & ,02093 & ,0093 &, 01751 & $>0,05$ \\
\hline Respuesta a la carga a apoyo medio &,- 0067 & 01877 & ,0040 & ,02028 &,- 0087 & ,01246 & $>0,05$ \\
\hline Apoyo medio a apoyo final &,- 0273 & ,01981 &,- 0260 & ,01404 &,- 0220 & 02210 & $>0,05$ \\
\hline Apoyo final a prebalanceo &,- 0235 &, 03955 &,- 0337 &, 03460 &,- 0303 &, 06830 & $>0,05$ \\
\hline Balanceo inicial a balanceo medio &, 0507 & ,02963 &, 0570 & 02411 &, 0510 & ,05015 & $0.02^{*}$ \\
\hline Balanceo medio a balanceo final & ,0533 & ,02526 & 0353 & 02973 & ,0420 & ,02178 & $>0,05$ \\
\hline
\end{tabular}

*Sig. Significancia estadística menor a 0,05. Probado a través de pruebas para medidas repetidas de Bonferroni.

\section{Discusión}

El propósito de este estudio fue determinar la diferencia entre los rangos de movimiento, velocidad y aceleración angular en mujeres con artrosis de rodilla con diferentes descargas de peso. Al realizar la comparación entre los rangos de movimiento encontrados en promedio en los tres momentos de evaluación y los referenciados según O'Sullivan (8). En el tobillo, el promedio es de 0 grados, valor que se encuentra dentro de los rangos normales; sin embargo, al realizar el análisis de comparación entre los tres grupos, se encuentra que la diferencia entre el $100 \%$ y el $80 \%$ del peso corporal es estadísticamente significativa con una diferencia de $3,2^{\circ}$ de plantiflexión mostrándose un tobillo en neutro con el $80 \%$ y $3^{\circ}$ de plantiflexion con el $100 \%$ del peso, siendo este último un contacto inicial más eficiente.

En la fase de respuesta a la carga en la articulación de la cadera se pasa de $30^{\circ}$ de flexión a $5^{\circ}$, dato que cabe dentro de estos rangos descritos. Por su parte, la articulación de la rodilla en la fase en mención pasa de $15^{\circ}$ a $5^{\circ}$, según lo reportado en el presente documento en promedio $18^{\circ}$ de flexión, dato cercano al patrón de marcha normal, esto en los tres momentos de medición. Con respecto al tobillo, es claro que no responde a los grados de movimiento referidos pues el promedio muestra de $2^{\circ}$ a $5^{\circ}$ de dorsiflexión y los grados descritos son de $10^{\circ}$ de dorsiflexión. 
En el apoyo medio, se encontró la cadera de $3^{\circ}$ a $10^{\circ}$ de flexión; sin embargo, en esta fase de la marcha se debería encontrar en neutro, pero al realizar la comparación entre los tres momentos de evaluación es evidente que no existen diferencias significativas, situación similar con la rodilla.

Las modificaciones con respecto a los rangos de movimiento en pacientes con artrosis de rodilla son diferentes, sobre todo en flexión de rodilla en cada fase de la marcha, esto como mecanismo de protección contra el dolor, ya que, si la flexión aumenta, el movimiento interarticular probablemente también lo hace incrementando el roce articular y así los síntomas (9). Sin embargo, los cambios en la excursión de movimiento dependen del estadio de la enfermedad, índice de masa corporal, edad y género, según Ko los rangos de movimiento no son estadísticamente diferentes en el plano sagital en las articulaciones de rodilla, tobillo y cadera entre personas sanas y con artrosis de rodilla en sus estadios 1 y 2 , esto comparando la marcha usual, la marcha rápida y después de 30 minutos (10).

Pero, además de lo anterior, el factor que modifica el comportamiento de la movilidad en el plano sagital es la presencia de dolor pues como lo afirma Maly, el comportamiento de la flexión de la rodilla tiene una relación con la presencia del principal síntoma que limita funcionalmente a las personas con esta condición de salud (11).

Para la articulación del tobillo se referencia que el momento en que más predomina la flexión plantar es en el prebalanceo y, a su vez, el de mayor flexión dorsal es el de respuesta a la carga; sin embargo, en las personas con osteoartritis de rodilla estos grados de movimiento disminuyen en cierta medida, pues los tiempos de la marcha son menores, lo que coincide con lo mostrado en el presente estudio, pues al poner a las participantes con el $100 \%$ del peso corporal los movimientos en el plano sagital de la articulación del tobillo fueron menores, en comparación con la carga del $80 \%$ y el $100 \%$ del peso (12).

No se comprobaron cambios demostrativos en el dolor de la rodilla en las pacientes examinadas, pues la diferencia de promedios antes, durante y después en los tres momentos de medición no fue estadísticamente significativa $(\dot{\mathrm{x}}=3,0 \neq$ $p>0,05)$, situación presumiblemente explicada sobre el hecho que sustenta que los pacientes que padecen dolor articular por artrosis en la rodilla y obesidad o sobrepeso podrían tener un cambio en la sintomatología si el peso corporal disminuye de forma sustancial y, así mismo, la carga sobre la rodilla, hecho que se consigue con la práctica habitual de ejercicio físico, alimentación saludable, actividades que contribuirán con el mejoramiento de las cualidades físicas de la persona que propenderán por la disminución de peso, la consiguiente baja de carga sobre la rodilla y así una reducción del dolor (13). Adicionalmente, para que se consigan cambios en el dolor se ha afirmado que es necesario el entrenamiento funcional progresivo en fuerza de resistencia para los músculos de cadera, rodilla y tobillo, con intensidad moderada, sumado a una dieta adecuada que mejore el peso corporal (14).

No se encontraron diferencias estadísticamente significativas entre la velocidad, aceleración, y rangos de movimiento frente a las tres descargas de peso en que fueron medidas las pacientes; sin embargo, otros estudios desarrollados en pacientes con trauma raquimedular afirman que posterior a controlar la descarga de peso en la marcha sí se modifican las variables de la marcha y, adicionalmente, favorece el entrenamiento propioceptivo de los pacientes (15).

Es conocido que la velocidad es una variable cinemática de la marcha que se asocia directamente con la presencia de artrosis de rodilla y los cambios radiográficos en la articulación afectada, se correlacionan con la disminución de la velocidad angular en la flexo-extensión de rodilla 
y con la velocidad lineal de desplazamiento en la marcha (16); sumado a esto, se afirma que la disminución de la velocidad se relaciona directamente con la severidad de la afección (17).

La velocidad lineal de desplazamiento durante la marcha en el presente estudio fue dada por la plataforma de rehabilitación locomotora $3 \mathrm{~km} / \mathrm{h}$ por lo cual no se midieron los cambios de esta variable con las diferentes cargas de peso; a pesar de esto, la velocidad angular se midió sin encontrarse diferencias significativas; no obstante, algunas investigaciones afirman que la complejidad de la marcha se reduce si la velocidad de esta es diferente de la velocidad autoseleccionada. Se infiere que esta disminución de la variabilidad de los parámetros angulares (velocidad y aceleración angular) sobre las articulaciones afectadas se produce por la falta de flexibilidad, propiocepción y fuerza muscular, lo que genera un patrón de marcha más lento en términos lineales y angulares (18).

La reducción en la velocidad de la marcha conduce a cambios en tres parámetros dimen- sionales, cinemáticos y cinéticos, está asociada con la reducción de la longitud del paso, cadencia, rangos de movimiento, fuerzas de reacción del suelo, en la cadera y la rodilla. Además, se ha conocido que el índice de masa corporal tiene un efecto sobre la biomecánica articular de la rodilla y su velocidad angular (19). Por lo anterior, se puede afirmar que los pacientes que presentan artrosis de rodilla y también un índice de masa corporal por encima del saludable pueden disminuir los parámetros cinemáticos de la marcha como la velocidad y la aceleración lineales y angulares, situación dada por las modificaciones en las diferentes fases de la marcha.

\section{Agradecimientos y conflicto de intereses}

Los autores agradecen a la Universidad de Boyacá y a la Universidad Santo Tomás de Tunja por facilitar los procesos y declaran no tener conflicto de intereses.

\section{Bibliografía}

1. Thomas A, Eichenberger G, Kempton C, Pape D, York S, Decker AM, Kohia M. Recommendations for the treatment of knee osteoarthritis, using various therapy techniques, based on categorizations of a literature review. J Geriatr Phys Ther. 2009;32(1):33-8.

2. Morgado I, Pérez AC, Moguel M, Pérez-Bustamante FJ, Torres LM. Guía de manejo clínico en la artrosis de cadera y rodilla. Rev Soc Esp Dolor. 2005;12(5):289-302.

3. Debi R, Mor A, Segal O, Segal G, Debbi E, Agar G, et al. Differences in gait patterns, pain, function and quality of life between males and females with knee osteoarthritis: a clinical trial. BMC Musculoskeletal Disord. 2009;10:127.

4. Elbaz A, Mor A, Segal G, Debbi E, Haim A, Halperin N, et al.. APOS therapy improves clinical measurements and gait in patients with knee osteoarthritis. Clin Biomech (Bristol, Avon). 2010;25(9):920-5.

5. Henriksen M, Graven-Nielsen T, Aaboe J, Andriacchi TP, Bliddal H. Gait changes in patients with knee osteoarthritis are replicated by experimental knee pain. Arthritis Care Res. 2010;62(4):501-9.

6. Brinkmann JR, Parry J. Rate and range of knee motion during ambulation in healthy and arthritic subjects. Phys Ther. 1985;65(7):1055-60.

7. Gök H, Ergin S, Yavuzer G. Kinetic and kinematic characteristics of gait in patients with medial knee arthrosis. Acta Orthop Scand. 2002;73(6):647-52. 
8. O'Sullivan S, Schmitz T. Physical Rehabilitation. $5^{\text {th ed }}$ Philadelphia: F. A. Davis Company; 2007.

9. Çelik F, Göçmez C, Karaman H, Kamaşak K, Kaplan İ, Akıl E, et al. Therapeutic effects of thymoquinone in a model of neuropathic pain. Current Therapeutic Research. 2014;76:11-6.

10. Ko SU, Ling SM, Schreiber C, Nesbitt M, Ferrucci L. Gait patterns during different walking conditions in older adults with and without knee osteoarthritis-Results from the Baltimore Longitudinal Study of Aging. Gait Posture. 2011; 33(2):205-10.

11. Maly MR, Costigan PA, Olney SJ. Mechanical factors relate to pain in knee osteoarthritis. Clin Biomech (Bristol, Avon). 2008;7;23(6):796-805.

12. Dumas R, Cheze L. Hip and knee joints are more stabilized than driven during the stance phase of gait: an analysis of the 3D angle between joint moment and joint angular velocity. Gait Posture. 2008;28(2):243-50.

13. Foy C G, Lewis C E, Hairston K G, Miller G D, Lang W, Jakicic J M, et al. Intensive lifestyle intervention improves physical function among obese adults with knee pain: findings from the Look AHEAD Trial. Obesity (Silver Spring). 2011;19(1):83-93.

14. Baker KR, Nelson ME, Felson DT, Layne JE, Sarno R, Roubenoff R. The efficacy of home based progressive strength training in older adults with knee osteoarthritis: a randomized controlled trial. J Rheumatol. 2001;28(7):1655-65.

15. Lucareli PR, Lima MO, Lima FP, de Almeida JG, Brech GC, D'Andréa Greve, JM. Gait analysis following treadmill training with body weight support versus conventional physical therapy: a prospective randomized controlled single blind study. Spinal Cord. 2011;49(9):1001-7.

16. Purser JL, Golightly YM, Feng Q, Helmick CG, Renner JB, Jordan JM. Association of slower walking speed with incident knee osteoarthritis-related outcomes. Arthritis Care Res (Hoboken). 2012;64(7):1028-35.

17. McDaniel G, Renner JB, Sloane R, Kraus VB. Association of knee and ankle osteoarthritis with physical performance. Osteoarthritis Cartilage. 2011;19(6):634-8.

18. Kiss RM. Effect of severity of knee osteoarthritis on the variability of gait parameters. J Electromyogr Kinesiol. 2011;21(5):695-703.

19. Sheehan KJ, Gormley J. The influence of excess body mass on adult gait. Clin Biomech. 2013;28(3):337-43. 